\title{
Degranulation of mast cells in dengue patients
}

L.V.S. Asher*, A.S. Norton*, S. Krivda**, H.K. Wong*, M.P. Mammen*, A.G. Lyons*, S. Thomas**, W. Sun*, K.H. Eckels*, D.W. Vaughn*.

*Walter Reed Army Institute of Research, Silver Spring, Maryland 20910-7500

**Department of Medicine, Walter Reed Army Medical Center, Washington DC 20307.

Dengue fever (DF) is an incapacitating illness with high fever, myalgia, frontal headache and a maculopapular rash. It has been suggested that rash develops as a result of endothelial cell damage due to viral replication in these cells. However, viral particles consistent with dengue virus have not been observed in the skin of dengue patients, and the only pathologic changes in small dermal blood vessels described so far, were gap formations between endothelial cells that could explain increased permeability of capillaries with subsequent edema [1]. Dengue viral antigen was detected by immunohistochemistry in Langerhans cells of one human volunteer [2].

Dengue is a "re-emerging" viral infection that has had an impact on U.S. soldiers during recent deployments. There is urgent need for an effective vaccine. However, non-human primates do not develop disease, and there is no in vitro correlate of protective immunity. Therefore, a human challenge model provides the only way to test the efficacy of dengue vaccines.

We examined skin biopsies from five volunteers who developed clinical signs of dengue infection and skin rash following inoculation with dengue virus. A $5 \mathrm{~mm}$ skin biopsy was obtained before inoculation, 4 days after (at the site of inoculation) and at the time of rash development. Biopsies were fixed in $4 \mathrm{~F} 1 \mathrm{G}$ ( $4 \%$ formaldehyde, $1 \%$ glutaraldehyde) and embedded in Epon. Thin sections were examined in a LEO $912 \mathrm{AB}$ electron microscope.

There was no evidence of dengue viral replication either in the epidermis (Langerhans cells) or in dermis. While extensive mononuclear perivascular inflammation was present in dermis 4 days after inoculation, only minor lymphocytic infiltrate was present at the time of skin rash. The most significant histological finding was extensive degranulation of mast cells located around small blood vessels in the skin biopsies obtained at the time of rash. As one of the major chemical mediators in mast cell granules is histamine that causes dilation of capillaries, release of histamine from mast cell granules might be responsible for rash and capillary permeability in dengue patients.

\section{References}

[1] S Sahaphong, et al. Southwest Asian J Trop Med Public Health 1980 Jun; 11 (2): 194-204.

[2] SJ Wu et al. Nat. Med. 2000 Jul; 6(7): 816-20. 

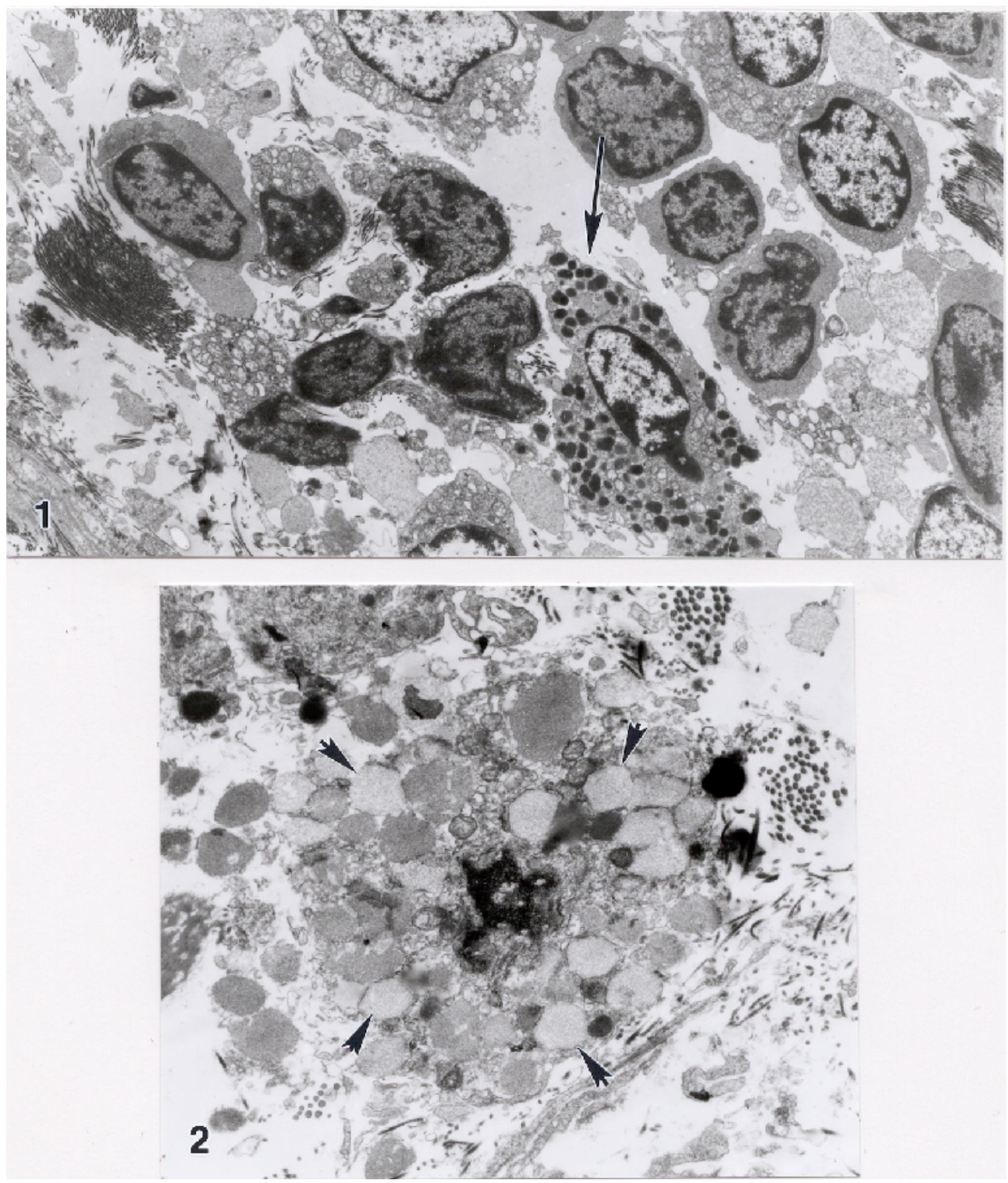

Fig.1. Mononuclear inflammatory infiltrate in the skin at the site of dengue inoculation, day 4 post-inoculation. Mast cell is normal (arrow). X 5,400

Fig. 2. Degranulation of mast cells at the time of skin rash (arrows), day 8 post-inoculation. $\mathrm{X} 12,600$ 